\title{
Business Intelligence para apoio à gestão na construção civil: uma revisão sistemática da literatura
}

\section{Business Intelligence to support management in civil construction: a systematic literature review}

\author{
Anderson Brunheira Lopes ${ }^{1}$, Clodis Boscarioli ${ }^{2}$, Eliane Nascimento Pereira ${ }^{3}$, Renata Camacho Bezerra ${ }^{4}$ \\ ${ }^{1}$ Universidade Estadual do Oeste do Paraná, Cascavel, Paraná, Brasil. ORCID: https://orcid.org/0000-0002-2217-2896 \\ 2 Universidade Estadual do Oeste do Paraná, Cascavel, Paraná, Brasil. ORCID: https://orcid.org/0000-0002-7110-2026 \\ 3 Universidade Estadual do Oeste do Paraná, Cascavel, Paraná, Brasil. ORCID: https://orcid.org/0000-0003-4285-3215 \\ ${ }^{4}$ Universidade Estadual do Oeste do Paraná, Cascavel, Paraná, Brasil. ORCID: https://orcid.org/0000-0002-4461-8473
}

Autor para correspondência/Mail to: Anderson Brunheira Lopes, andersonblopes@bol.com.br

Recebido/Submitted: 03 de maio de 2020; Aceito/Approved: 02 de julho de 2020

\begin{abstract}
Copyright (C) 2020 Lopes, Boscarioli, Pereira \& Bezerra. Todo o conteúdo da Revista (incluindo-se instruções, política editorial e modelos) está sob uma licença Creative Commons Atribuição-NãoComercial-Compartilhalgual 3.0 Não Adaptada. Ao serem publicados por esta Revista, os artigos são de livre uso em ambientes educacionais, de pesquisa e não comerciais, com atribuição de autoria obrigatória. Mais informações em http://revistas.ufpr.br/atoz/about/submissions\#copyrightNotice.
\end{abstract}

\begin{abstract}
Resumo
Introdução: introdução: A gestão é essencial para que se cumpram os requisitos de um projeto, e as ferramentas computacionais de Business Intelligence (BI) têm grande potencial de contribuição, fornecendo informações gerenciais sobre o negócio. Ferramentas desse tipo são utilizadas em diversos setores da indústria, porém na construção civil, foco deste trabalho, o cenário é diferente, com muito a avançar. Diante disso, apresenta-se um levantamento das ferramentas de BI aplicáveis ao setor da construção e suas utilizações.

Metodologia: conduz uma revisão sistemática da literatura, que analisou 595 artigos de seis bases de dados (ACM, Engineering Village, IEEE, Material Science Engineering, Science Direct, Scopus e Web of Science).

Resultados: identifica 12 diferentes aplicações, principalmente na área de gestão de custos, orçamento da obra e segurança do trabalho. Nas aplicações, foram evidenciadas utilizações das tecnologias de Data Warehouse e OLAP. Verifica que a maioria das ferramentas de BI foram desenvolvidas para cada empresa em detrimento dos softwares comerciais.

Conclusão: existem diversas ferramentas de BI para a construção civil, com diferentes aplicações. A maioria dos softwares foram desenvolvidos para cada caso estudado devido às características únicas do setor da construção. A adoção em larga escala das ferramentas passe pela cooperação entre empresas, entidades de classe e universidades. Verifica limitações na pesquisa quanto à caracterização das empresas, devido à ausência desses dados nos artigos analisados. Sugere que os desafios de implementação das tecnologias e as limitações verificadas sejam abordados em estudos futuros.
\end{abstract}

Palavras-chave: Business Intelligence; Ferramentas Computacionais; Análise de dados; Obras de Edificações; Revisão Sistemática da Literatura.

\begin{abstract}
Introduction: Management is essential for meeting the requirements of a project and the computational tools of Business Intelligence (BI) have great potential to contribution, providing management information about the business. Tools of this type are used in several sectors of the industry, but in civil construction, the focus of this work, the scenario is different, with much to aadvance. Therefore, a survey of BI tools applicable to the construction sector and its uses ar presented.

Method: it conducts a systematic review of the literature, which analyzed 595 articles from six databases (ACM, Engineering Village, IEEE, Material Science Engineering, Science Direct, Scopus and Web of Science).

Results: it identifies 12 different applications, mainly in cost management, budget and job security. In the applications, uses of Data Warehouse and OLAP technologies were evidenced. In addition, it was found that most BI tools were developed for each company to the detriment of commercial software. Conclusions: there are several BI tools for civil construction, with different purposes. Most softwares were developed for each case studied due to the unique characteristics of the construction sector. It is believed that the large-scale adoption of the tools involves cooperation between companies, class entities and universities. Limitations were found in the research regarding the characterization of companies, due to the absence of this data in the analyzed articles. Finally, it is suggested that the challenges of implementing the technologies and the verified limitations can be addressed in future studies.
\end{abstract}

Keywords: Business Intelligence; Computational Tools; Data Analysis; Building Works; Systematic Literature Review.

\section{INTRODUÇÃO}

A construção civil possui relevância para a economia nacional, sendo a receita proveniente desse setor, segundo o Instituto Brasilero de Geografia e Estatística (2020), correspondente a aproximadamente $25 \%$ de toda a receita gerada pela indústria brasileira. Além disso, aproximadamente 6,5 milhões de brasileiros trabalharam em atividades da construção no primeiro trimestre de 2018 (Instituto Brasilero de Geografia e Estatística, 2020). Esse número corresponde a 7,2\% da população total ativa desse período.

As empresas da construção civil e as dos demais setores da indústria possuem uma lacuna considerável na busca por novas tecnologias e aprimoramento de seus princípios gerenciais. É pertinente, portanto, uma análise dos 
potenciais benefícios obtidos com a implantação de tecnologias que possam melhorar o desempenho dos processos de gestão dessas empresas e que podem influenciar na expansão no mercado.

Existem diversos softwares de apoio à gestão de processos, sendo os mais difundidos os da categoria Enterprise Resource Planning (ERP). Esses sistemas, comumente encontrados desde empresas de varejo até grandes escritórios, apoiam todo o ciclo de vida de um processo de negócio, como controle de estoques, faturamento, emissão de notas e cadastro de clientes. Além da questão operacional, esses sistemas mantém importantes registros das operações, porém não fornecem dados de apoio à decisão estratégica, visto que não possuem ferramentas de Business Intelligence (BI). Por outro lado, os registros neles armazenados podem ser utilizados por ferramentas mais modernas, tornando-os úteis para o processo decisório.

Buscando preencher essa lacuna, surgiram os softwares baseados em conceitos de Data Warehouse (DW) e/ou Online Analytical Processing (OLAP), que geralmente são enquadrados na categoria de sistemas de BI, que são, segundo Chen, Chiang, e Storey (2012), ferramentas de apoio à tomada de decisões com base em informações certas, na hora certa. Esses softwares fornecem, por meio de Dashboards e outras interfaces de visualização, informações sintetizadas que podem auxiliar o gestor a acompanhar indicadores do negócio.

Existem diversos softwares de BI com essas características, que podem ser utilizados sem agregar custos às empresas e existem ainda versões gratuitas de softwares comerciais. A tecnologia conhecida como Data Warehouse é um dos pilares do conceito de BI. Em uma definição simples, podemos descrever essa tecnologia como um repositório que armazena dados pré-processados e não voláteis, provenientes de múltiplas - e provavelmente heterogêneas - bases de dados Chau, Cao, Anson, e Zhang (2003).

Esse repositório, que não descarta dados antigos, é utilizado como fonte de dados para a tecnologia OLAP, que conta com interface intuitiva, onde o usuário pode observar os dados por diversas perspectivas, tendo maiores chances de obter insights para tomar melhores decisões baseadas em informações (Ahmad, Azhar, \& Lukauskis, 2004). OLAP pode ser definido ainda como o conjunto de tecnologias que possibilitam o rápido processamento de dados, apresentando-os geralmente em forma de um cubo multidimensional (Konikov, Kulikova, \& Stifeeva, 2018).

Segundo Girsang et al. (2018), BI pode ser descrita como a capacidade de processar dados previamente armazenados, com o intuito de prover informações de negócios que possam trazer benefícios ao processo decisório; e, segundo Muntean e Surcel (2013), BI utiliza Extraction, Transform and Load (ETL) para extrair dados de diversas fontes, transformá-los e efetuar a carga em Data Warehouses de modo a gerar relatórios, ferramentas de consulta ad-hoc, análises estatísticas, OLAP, Dashboards e scorecards.

Com isso, conhecidos os desafios a serem enfrentados pelas empresas de construção civil na adoção de novas tecnologias para aprimoramento de seus princípios gerenciais, bem como o grande potencial de contribuição das ferramentas de BI e considerando a falta de pesquisas sobre o tema, esta pesquisa buscou exemplos da utilização de ferramentas tecnológicas na literatura, de forma a apresentar alternativas para a área da construção civil.

Para isso, uma revisão sistemática da literatura (RSL) foi realizada, buscando respostas sobre a utilização de conceitos de Data Warehouse e OLAP no âmbito do conjunto de tecnologias pertencentes à área de BI, no apoio a processos gerenciais da construção civil. A RSL almejou respostas para duas perguntas, sendo elas: (1) quais softwares/tecnologias de BI são utilizados no setor da construção civil; e (2) com que finalidade foram utilizadas.

Este artigo está estruturado em quatro seções, sendo a primeira delas a Introdução, em que foram apresentados a motivação para a elaboração do trabalho, o problema em estudo e um breve referencial teórico sobre o tema. A segunda seção, chamada de Metodologia, apresenta o protocolo da RSL e suas particularidades; enquanto a terceira seção, intitulada Resultados, reúne os dados extraídos dos estudos primários identificados, em busca das respostas às perguntas definidas. Por fim, na seção Conclusões, são apresentadas as considerações finais e as limitações da pesquisa e propostas para trabalhos futuros.

\section{METODOLOGIA}

Para Kitchenham (2007), uma Revisão Sistemática da Literatura é uma forma de identificar e interpretar, com base em parâmetros pré-definidos, todos os estudos concernentes a determinado tema. Os estudos individuais avaliados em uma RSL são chamados de estudos primários, tornando a RSL um estudo secundário sobre o assunto.

Na área de gestão, a revisão da literatura é uma ferramenta essencial, utilizada para gerenciar a diversidade de conhecimentos e guiar uma investigação acadêmica específica (Tranfield, Denyer, \& Smart, 2003). Uma revisão efetiva cria uma base sólida para o avanço do conhecimento, facilita o desenvolvimento de teorias, limita áreas na qual existem pesquisas em abundância e revela áreas em que pesquisas são necessárias (Chen, Chiang, \& Storey, 2002). 
Tranfield et al. (2003) e Kitchenham (2007) descrevem a estrutura de uma RSL em três passos principais, sendo eles (i) Planejamento; (ii) Condução; e (iii) Relato. Esta pesquisa também adotou essa estruturação, apresentada em detalhes a seguir.

\section{Planejamento da Revisão Sistemática}

Segundo Kitchenham (2007), o planejamento de uma RSL é composto pela execução de: (i) Identificação da necessidade de uma RSL; (ii) Comissionamento da RSL; (iii) Definição das perguntas de pesquisa; (iv) Desenvolvimento do protocolo; e (v) Validação do protocolo. A seguir, apresentam-se os itens (i), (iii) e (iv), considerados os mais relevantes para o entendimento do trabalho.

Quanto à necessidade de elaboração da RLS, em buscas preliminares efetuadas nas bases de dados, não foram encontradas revisões que abordassem direta ou indiretamente o tema estudado na presente pesquisa. Com isso, decidiu-se elaborar esta revisão sistemática, com o objetivo de se abordar e sumarizar todos os artigos que tratam sobre o tema.

Além disso, busca-se reunir essas informações para que outros autores possam conduzir suas pesquisas a partir do presente estudo. A metodologia da RSL busca evidenciar, de forma clara, os passos e critérios definidos na condução da revisão sistemática. Assumindo a necessidade de execução de uma RSL, foram definidas duas perguntas a serem por ela respondidas:

1 Quais softwares/tecnologias de Business Intelligence são utilizados na construção civil?

O propósito dessa pergunta foi o de identificar, em primeiro lugar, se existem softwares/tecnologias de BI utilizados no setor da construção civil para então, caso existam, listá-los.

2 Como e com que finalidade os softwares/tecnologias foram utilizados?

Identificados os softwares e tecnologias, a presente pergunta busca elucidar em que etapa do processo de gestão eles são aplicados e de que forma são utilizados.

\section{Desenvolvimento do Protocolo RSL}

O protocolo é a etapa que documenta a metodologia a ser utilizada na condução da RSL e segundo Kitchenham (2007), é composto por (i) Definição das bases de dados; (ii) Definição da estratégia de pesquisa; (iii) Definição dos critérios de seleção dos estudos primários; (iv) Definição do método de extração de informações; e (v) Definição da forma de apresentação dos dados. Para condução da revisão foi utilizado o software StArt, que fornece apoio desde a definição do protocolo até a extração e análise dos resultados.

Alguns critérios foram definidos para a escolha das bases de dados, sendo estes: Contém estudos relacionados à Tecnologia da Informação (TI), gestão, tecnologia, construção civil e que permita busca com operadores booleanos. Com esses critérios, foram definidas como bases de dados os seguintes repositórios: 1. ACM Digital Library; 2. Engineering Village; 3. IEEE Xplore Digital Library; 4. Material Science Engineering; 5. Science Direct; 6. SScopus; e 7 . Web of Science.

Com essas bases de dados, acredita-se ter sido possível maximizar as chances de encontrar estudos primários. Além disso, são bases conhecidas e difundidas na comunidade científica. Como estratégia de pesquisa, foi construída uma string de busca com operadores booleanos. Como não é possível utilizar a mesma string em todas as bases, pois estas possuem engines de busca avançada com diferentes características, foram formuladas, então, sete strings, adaptadas para cada uma das bases de dados. Abaixo, apresenta-se a string padrão utilizada:

\section{("Data Warehouse" OR "OLAP" OR "Business Intelligence") AND ("construction management" OR "Construction Planning" OR "construction enterprises" OR "construction work" OR "construction works" OR "building construction" OR "construction company").}

A RSL foi conduzida levando em consideração artigos científicos publicados a partir do ano 2000; com isso, o período de abrangência da pesquisa compreende aproximadamente 19 anos, visto que a aplicação das strings ocorreu no mês de março de 2019. Importante ressaltar que, na área de tecnologia, estudos com mais de cinco anos são considerados obsoletos, porém destaca-se que, no presente trabalho, foram levados em consideração tecnologias lançadas há muitos anos e utilizadas até hoje, como Data Warehouse, OLAP e BI. Além disso, com a amplitude de tempo, poderão ser observadas as tendências de desenvolvimento tecnológico sobre o tema no decorrer dos anos.

Como estratégia de seleção dos estudos primários, foi definido um conjunto de critérios de inclusão e exclusão, baseados no escopo da pesquisa, de forma a garantir que somente estudos referentes ao assunto da RSL fossem escolhidos. Para ser incluído, o estudo precisava atender um ou mais dos critérios de inclusão. Para ser desconsiderado, precisava atender qualquer um dos critérios de exclusão. A seguir, apresentam-se os critérios de inclusão e exclusão, nos Quadros 1 e 2, respectivamente. 


\section{Critérios de inclusão}

I1 - O artigo trata de Business Intelligence e Construção Civil diretamente e não apenas menciona no texto; 12 - O artigo trata de OLAP e Construção Civil diretamente e não apenas menciona no texto; 13 - O artigo trata de Data Warehouse e Construção Civil diretamente e não apenas menciona no texto.

Quadro 1. Critérios de inclusão.

Fonte: Elaborado pelos autores (2020).

\begin{tabular}{|l|}
\hline Critérios de exclusão \\
\hline E1 - O artigo não está totalmente disponível online; \\
\hline E2 - O artigo não está redigido completamente no idioma inglês ou português; \\
\hline E3 - O artigo não relacionado à área da construção civil; \\
\hline E4 - O artigo trata de softwares/tecnologias de BI, porém não está relacionado à gestão na construção civil; \\
\hline E5 - O artigo não trata de BI na construção civil; \\
\hline $\begin{array}{l}\text { E6 - O documento não é um artigo, sendo na verdade um relato técnico, livro ou capítulo de livro, procedi- } \\
\text { mento, prefácio ou editorial. }\end{array}$ \\
\hline
\end{tabular}

Quadro 2. Critérios de exclusão.

Fonte: Elaborado pelos autores (2019).

\section{Execução da Revisão Sistemática}

A presente seção apresenta os principais passos executados na condução da RSL. Serão apresentadas as seguintes etapas: (i) Identificação e seleção dos estudos primários; (ii) Verificação da qualidade dos estudos primários; e (iii) Extração de dados e síntese dos estudos primários.

A identificação dos candidatos a estudos primários ocorreu com a aplicação das strings de busca nas engines de busca avançada das respectivas bases de dados. Nessa etapa, foram identificados 595 candidatos a estudos primários. Após a leitura dos títulos, resumos e palavras-chave dos trabalhos, foram aplicados os critérios de inclusão e exclusão pré-definidos, listados nos Quadros 1 e 2. Após essa etapa, foram rejeitados 520 estudos, 44 foram identificados como duplicados e 31 estudos foram aceitos.

Para a verificação da qualidade dos estudos primários, estabeleceram-se novos critérios de inclusão/exclusão, sendo estes aplicados após a leitura completa dos artigos. Permaneceram os artigos publicados revisados por pares e que respondessem ao menos uma das questões definidas. Além disso, excluíram-se artigos duplicados que não puderam ser identificados apenas com a leitura do título e do resumo, devido às pequenas alterações efetuadas pelos autores, imperceptíveis nas leituras dos títulos, resumos e palavras-chave. Após a aplicação dos critérios de verificação de qualidade dos estudos, restaram 23 artigos científicos. A Tabela 1 apresenta as quantidades de estudos de cada fase do processo de seleção.

\begin{tabular}{lccc}
\hline Base de dados & $\begin{array}{c}\text { Candidatos a estudos } \\
\text { primários }\end{array}$ & $\begin{array}{c}\text { Estudos primários } \\
\text { identificados }\end{array}$ & $\begin{array}{c}\text { Resultado após } \\
\text { avaliação de } \\
\text { qualidade }\end{array}$ \\
\hline Science Direct & 211 & 13 & 9 \\
ACM Digital Library & 183 & 0 & 0 \\
Material Science Engineering & 134 & 5 & 5 \\
Scopus & 30 & 8 & 8 \\
Engineering Village & 15 & 0 & 0 \\
Web of Science & 15 & 4 & 0 \\
IEEE Xplore Digital Library & 7 & 1 & 1 \\
Total & 595 & 31 & 23 \\
\hline
\end{tabular}

Tabela 1. Estudos primários selecionados.

Fonte: Elaborada pelos autores (2019).

Para fins de padronização da nomenclatura dos estudos e para a sua apresentação geral, a fim de permitir rastreabilidade aos dados, a seguir, nos Quadros 3 e 4, apresenta-se a lista com os estudos finais identificados em eventos e periódicos, respectivamente. Para estudos publicados em eventos, utilizou-se a codificação E01, E02 e assim sucessivamente. Para os estudos publicados em periódicos, foi adotada a codificação P01, P02 e assim por diante. 


\begin{tabular}{|c|c|l|}
\hline Ano & ID & Referência \\
\hline 2002 & E01 & (Cao, Chau, Anson, \& Zhang, 2002) \\
\hline 2005 & E02 & (Rujirayanyong \& Shi, 2005) \\
\hline 2007 & E03 & (Vuori, 2007) \\
\hline 2009 & E04 & (Wang, Xi, \& Gao, 2009) \\
\hline 2017 & E05 & (Montaser \& Montaser, 2017) \\
\hline 2018 & E06 & (Girsang et al., 2018) \\
\hline 2018 & E07 & (Konikov et al., 2018) \\
\hline 2018 & E08 & (Konikov, 2018) \\
\hline
\end{tabular}

Quadro 3. Estudos publicados em eventos. Fonte: Elaborado pelos autores (2019).

\begin{tabular}{|l|l|l|}
\hline Ano & ID & Referência \\
\hline 2001 & P01 & (Rezgui, 2001) \\
\hline 2003 & P02 & (Chau et al., 2003) \\
\hline 2004 & P03 & (Ahmad et al., 2004) \\
\hline 2005 & P04 & (Chau, Anson, \& Zhang, 2005) \\
\hline 2006 & P05 & (Rujirayanyong \& Shi, 2006) \\
\hline 2007 & P06 & (Moon, Kim, \& Kwon, 2007) \\
\hline 2010 & P07 & (Cheng, Lin, \& Leu, 2010) \\
\hline 2010 & P08 & (Szelka \& Wrona, 2010) \\
\hline 2011 & P09 & (Rezaei, Çelik, \& Baalousha, 2011) \\
\hline 2011 & P10 & (Z. Ma, Lu, \& Wu, 2011) \\
\hline 2013 & P11 & (L. Ma, Luo, Bin, \& Chen, 2013) \\
\hline 2013 & P12 & (Chong \& Phuah, 2013) \\
\hline 2013 & P13 & (Z. Ma, Lu, \& Wu, 2013) \\
\hline 2014 & P14 & (Hammad, AbouRizk, \& Mohamed, 2014) \\
\hline 2016 & P15 & (Martínez-Rojas, Marín, \& Miranda, 2016) \\
\hline
\end{tabular}

Quadro 4. Estudos publicados em periódicos. Fonte: Elaborado pelos autores (2019).

Concluídas as etapas anteriores, foram conduzidas leituras completas dos 23 artigos em busca de respostas às perguntas definidas. Como cada pergunta tem um objetivo, foram definidas estratégias distintas para cada pergunta de pesquisa. Para a Pergunta 1, por se tratar de pergunta que engloba duas questões, foram definidos dois atributos a serem verificados, sendo estes: qual a tecnologia de BI utilizada (Data Warehouse, OLAP ou ambas), bem como qual o software utilizado. Ao fim, foram listados todos os softwares/tecnologias identificados nos estudos. No caso da Pergunta 2, por se tratar de pergunta exploratória, a estratégia adotada para extração foi a de listagem das diversas formas de utilização dos softwares/tecnologias de BI na construção civil identificados nos estudos.

\section{RESULTADOS}

Na presente seção, serão apresentados os resultados obtidos com a execução da RSL. Inicialmente apresenta-se a Figura 1, que mostra os anos de publicação dos estudos primários identificados, em que se pode observar que aproximadamente $52 \%$ dos estudos foram publicados a partir de 2010. Tal fato pode ser justificado pela evolução dos dispositivos móveis, como smartphones e tablets, popularizados mundialmente dois anos antes (Gowthami \& Venkatakrishnakumar, 2016). Com essa evolução, a geração de dados aumentou em escala exponencial e, como uma das principais funções das tecnologias de BI é transformar dados em conhecimento, é natural que um crescimento do volume das informações disponíveis fomentasse maior interesse dos pesquisadores. Nota-se certa falta de regularidade nas publicações, porém é possível notar uma linha de tendência crescente, indicando que o tema vem sendo estudado com mais frequência atualmente. 


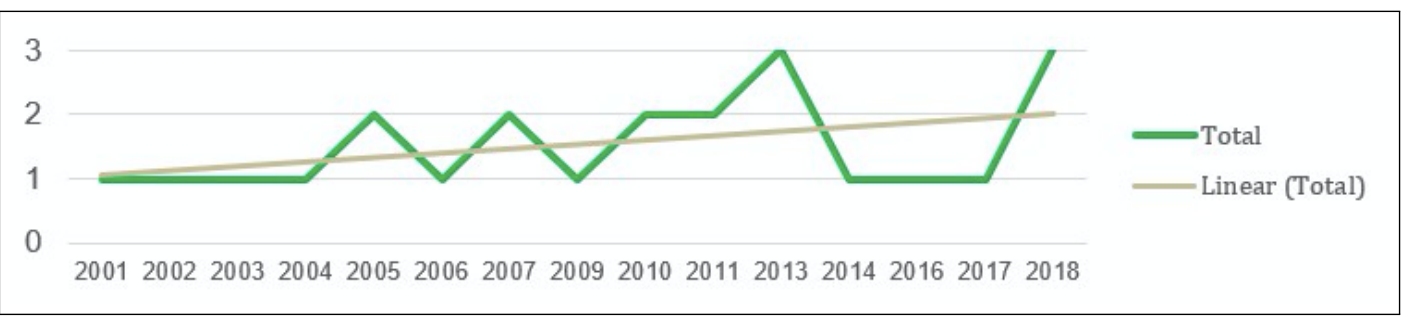

Figura 1. Estudos selecionados - por ano de publicação. Fonte: Dados da pesquisa (2019).

Já na apresentação das respostas da Pergunta 1, mostra-se a seguir o resultado da busca pelos softwares mencionados nos estudos. Após as investigações, observou-se que 12 dos 20 softwares mencionados foram desenvolvidos especificamente para o caso estudado, o que representa $60 \%$ das respostas. Em segundo lugar, aparece três vezes o software Primavera, que corresponde a 15\%; seguido pelo SAP, citado duas vezes, que equivale a 10\%; e Qlik, Pentaho e Statsoft Estatística, sendo relacionados uma vez, o que reflete $5 \%$ do total para cada um. O Quadro 5 apresenta as respostas fornecidas por cada estudo primário identificado.

\begin{tabular}{|l|l|}
\hline Softwares & Estudo ID \\
\hline Pentaho & E06 \\
\hline Primavera & E02; P05; P14 \\
\hline Qlik & E06 \\
\hline SAP & E04; P10 \\
\hline Software próprio & E01; P01; P02; P03; P04; P05; P06; P08; P09; P12; P13; P15 \\
\hline Statsoft Estatística & P07 \\
\hline
\end{tabular}

Quadro 5. Softwares identificados nos estudos primários.

Fonte: Elaborado pelos autores (2019).

Acredita-se que o grande número de soluções desenvolvidas especificamente para cada caso deve-se às características inerentes ao setor da construção civil, que é conhecido por envolver trabalhos em campo, em locais temporários e com grande produção de dados em diferentes formatos (Martínez-Rojas, Marin, \& Amparo Vila, 2012). Por outro lado, as tecnologias de BI foram desenvolvidas originalmente para fornecerem informações executivas (Negash \& Gray, 2003), o que justificaria a necessidade de desenvolvimento de soluções alternativas e adaptadas, visto que os softwares comerciais não teriam capacidade para atender completamente o setor da construção civil. No início dos anos 2000, Cao et al. (2002) afirmava que o fato das obras serem temporárias e específicas, ou seja, diferentes umas das outras, exigia diferentes formas de uso para as ferramentas de BI, propondo um uso com visão a curto prazo em detrimento do uso para níveis estratégicos de longo prazo como habitual.

Na linha dos softwares específicos, apresentam-se alguns exemplos encontrados, como o de Cao et al. (2002) que apresentou um software baseado nas tecnologias OLAP e DW para aprimorar a gestão de custos de obras, possibilitando o monitoramento em tempo real das quantidades de insumos adquiridos, por meio de informações gráficas e numéricas. Para isso, utilizou a modelagem multidimensional no esquema estrela para montar um DW com dados extraídos via Structured Query Language (SQL) do banco de dados do ERP existente na empresa. Com os dados armazenados no DW, utilizou a ferramenta OLAP para gerar as análises necessárias, possibilitando a rápida tomada de decisão gerencial.

Ainda utilizando DW e OLAP, Moon et al. (2007) propuseram uma ferramenta específica que estimava os custos das obras, ou seja, elaborava os orçamentos de obras futuras, com base em análises estatísticas de dados históricos de obras similares. Os autores obtiveram resultados importantes, porém ressaltaram que os resultados estão fortemente ligados ao grau de correlação entre as características das obras analisadas.

Utilizando as tecnologias de BI para a gestão de resíduos, Z. Ma et al. (2013) desenvolveram uma ferramenta em plataforma web, que reúne dados de geração de resíduos de diversas obras em um DW, permitindo a elaboração de estimativas das quantidades de resíduos a serem gerados em novas obras mediante consultas com OLAP.

Nesse sentido, quando analisadas as tecnologias de BI utilizadas nos estudos, foi possível observar qual era utilizada com mais frequência, sendo consideradas na listagem tecnologias de Data Warehouse e OLAP, utilizadas de forma individual ou em conjunto, sempre considerando o enfoque em Business Intelligence.

Foram identificados 22 registros de utilização de Data Warehouse, ou seja, em mais de $95 \%$ dos estudos foram verificadas aplicações com essa tecnologia. Verifica-se ainda que 15 registros relacionam a utilização das duas tecnologias em conjunto. Esse é o mesmo número de vezes que se constata a utilização da tecnologia OLAP. 
Com base no resultado acima e na amostra estudada, pode-se afirmar que a tecnologia OLAP não foi utilizada isoladamente, apenas em conjunto com o DW.

Para a Pergunta 2, apresenta-se na Figura 2 a existência de 12 diferentes aplicações dos softwares/tecnologias de BI para a construção civil, em diversos setores e fases do processo de execução da obra. Para a identificação dessas aplicações, foi necessária leitura e interpretação dos estudos analisados, com foco na metodologia adotada em cada um. Após as leituras, foram criados grandes grupos para categorização das aplicações. Cita-se como exemplo a categoria de "Elaboração do Orçamento", que inclui estudos em que se desenvolveram sistemas inteligentes para a elaboração de planilhas de preços de obras, sistemas de estimativas de custos de obras com base em análises estatísticas de dados de outras obras executadas, dentre outros. Com isso, ressalta-se que tais classificações estão suscetíveis à subjetividade dos autores, no entanto os resultados são passíveis de verificação por meio dos dados de rastreio fornecidos no presente trabalho.

Na área de elaboração dos orçamentos de obras, encontra-se o maior número de registros de utilização, sendo verificadas sete ocorrências, que correspondem a aproximadamente $27 \%$ do total; seguido por gestão de custos, com cinco registros, que equivale a aproximadamente $19 \%$.

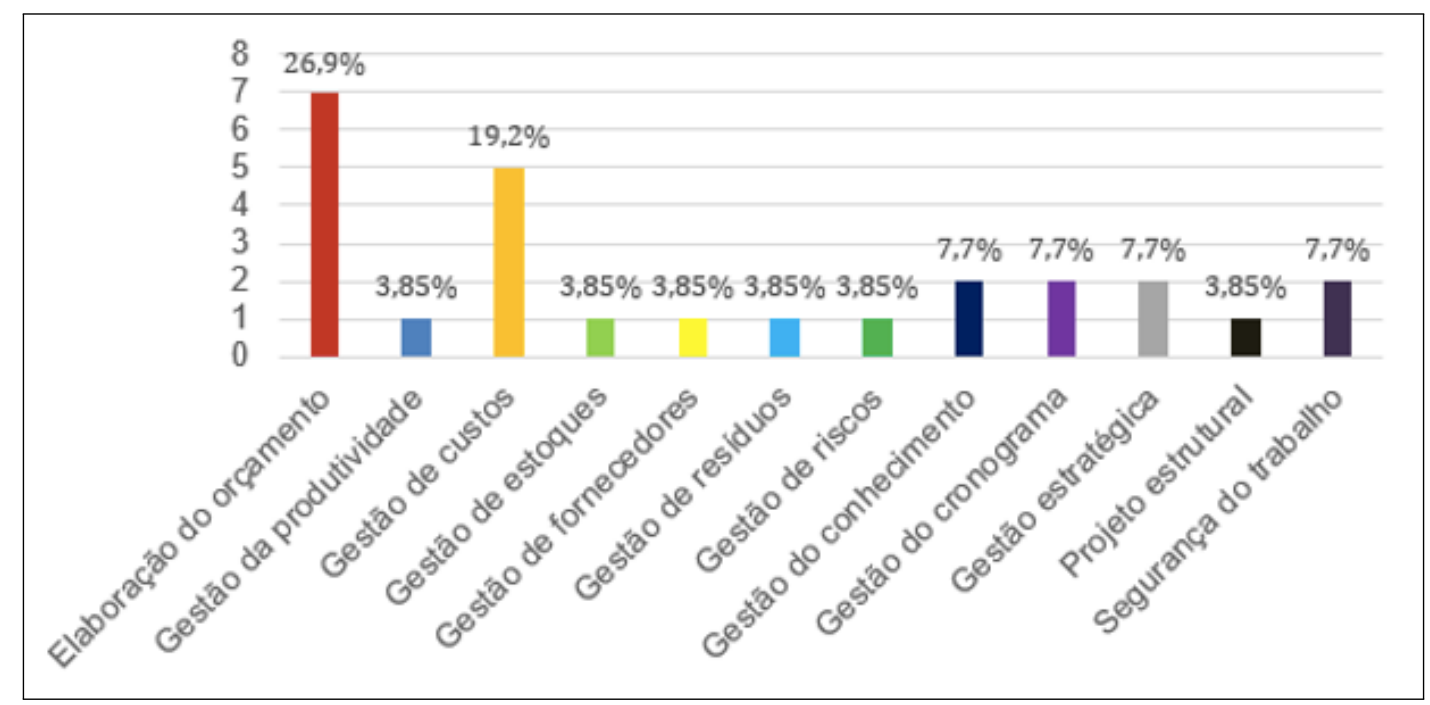

Figura 2. Aplicações de BI na construção civil. Fonte: Dados da pesquisa (2019).

Verificaram-se ainda aplicações em gestão do cronograma, gestão do conhecimento, gestão estratégica e em segurança do trabalho, sendo cada uma correspondente a aproximadamente 7,7\% do total. Por fim, com um registro cada, apresentam-se aplicações em gestão da produtividade, gestão de estoques, fornecedores, riscos e resíduos e, também, como apoio à decisão na elaboração de projetos estruturais, como pode ser visto no Quadro 6.

\begin{tabular}{|l|l|}
\hline Aplicação & Estudo ID \\
\hline Elaboração do orçamento & E02; P5; P06; P10; P14; P15; P12 \\
\hline Gestão da produtividade & P09 \\
\hline Gestão de custos & E01; E04; E06; P02; P12 \\
\hline Gestão de estoques & P05 \\
\hline Gestão de fornecedores & P12 \\
\hline Gestão de resíduos & P13 \\
\hline Gestão de riscos & P12 \\
\hline Gestão do conhecimento & P01; P12 \\
\hline Gestão do cronograma & E06; P04 \\
\hline Gestão estratégica & E03; P03 \\
\hline Projeto estrutural & P08 \\
\hline Segurança do trabalho & P07; P11 \\
\hline
\end{tabular}

Quadro 6. Aplicações identificadas nos estudos primários.

Fonte: Elaborado pelos autores (2019).

Em uma análise complementar, com o intuito de conhecer de forma mais aprofundada o modus operandi das empresas de construção civil que fazem uso de BI, foram relacionadas as aplicações e os tipos de softwares utilizados em cada uma delas (Figura 3). 


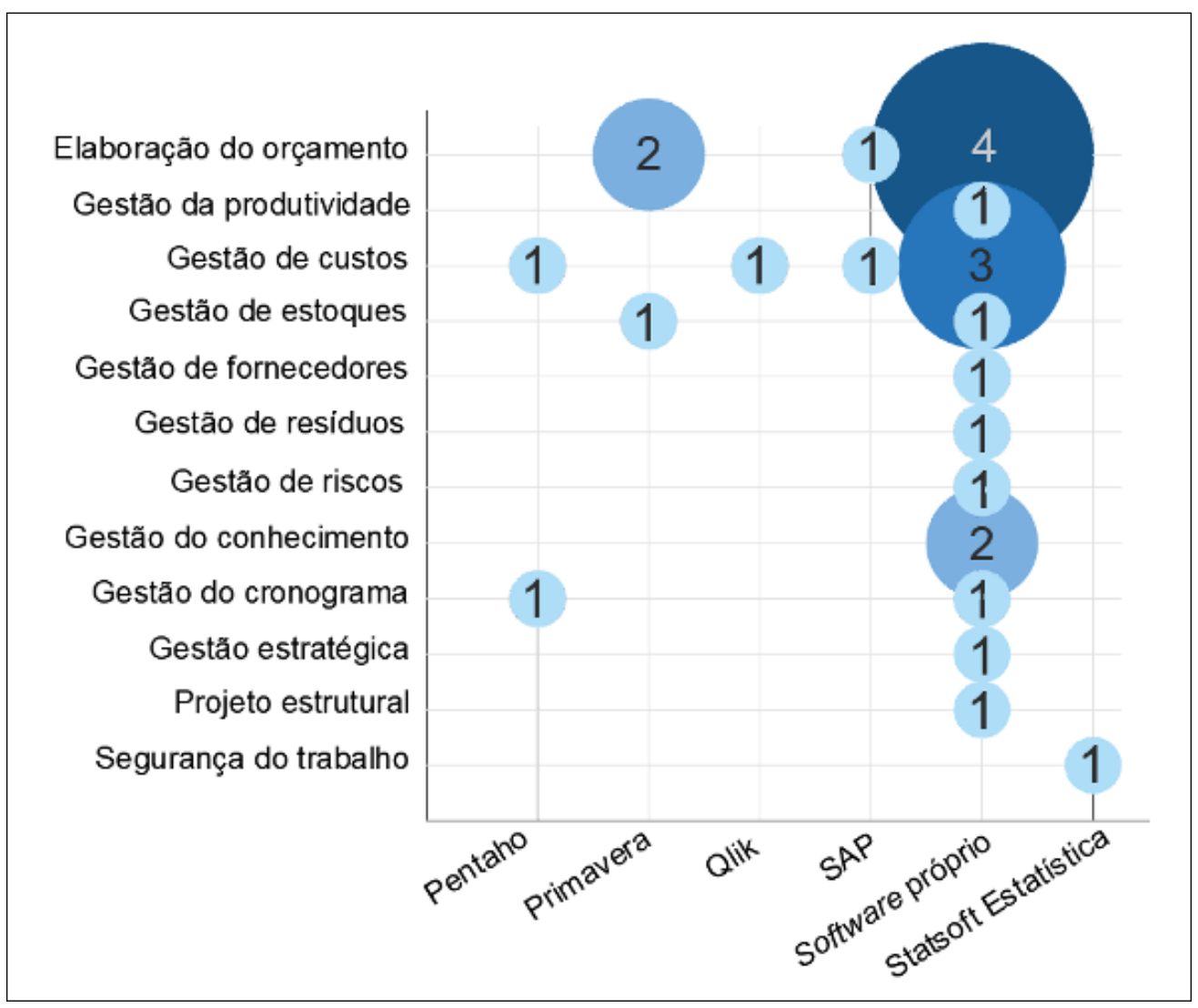

Figura 3. Relação entre aplicação e software utilizado.

Fonte: Dados da pesquisa (2019).

Pode-se observar que aplicações de elaboração do orçamento, com quatro cruzamentos, e gestão de custos, com três, foram implementadas por meio de softwares próprios. Em seguida, observa-se a utilização do software Primavera relacionada à elaboração de orçamentos, por duas vezes, e, para a gestão de estoques, por uma vez. Os softwares Pentaho e Qlik foram relacionados com aplicações de gestão do cronograma e do custo da obra. O SAP foi relacionado à elaboração de orçamento e gestão de custos; e o Statsoft Estatística foi aplicado na área de segurança do trabalho.

\section{CONCLUSÕES}

Para a Pergunta 1, que questionava "Quais softwares/tecnologias de Business Intelligence são utilizados na construção civil?", evidenciou-se a utilização dos softwares Pentaho, Primavera, Qlik, SAP e Statsoft Estatística, porém $60 \%$ dos softwares verificados não eram comerciais e haviam sido desenvolvidos especificamente para cada empresa. É possível que tal fato ocorra devido às características inerentes ao setor da construção civil, que é conhecido por envolver trabalhos em campo, em locais temporários e com grande geração e transferência de dados em diferentes formatos, como planilhas, especificações e projetos (Martínez-Rojas et al., 2016). Além disso, as tecnologias de BI foram desenvolvidas originalmente para fornecerem informações executivas e de apoio à decisão (Sapateiro \& Rui, 2019), o que justificaria a necessidade de desenvolvimento de soluções alternativas e adaptadas, projetadas para atender às necessidades decisórias de cada empresa individualmente, ainda que todas no mesmo domínio da aplicação.

Quanto às tecnologias, foi possível observar utilizações frequentes do Data Warehouse e OLAP, sendo que OLAP não foi utilizado sem associação ao Data Warehouse, o que já era esperado, pois o segundo utiliza dados previamente armazenados de forma relacional do primeiro, formando, assim, as tecnologias de BI.

Para a Pergunta 2 "Como e com que finalidade os softwares/tecnologias foram utilizados?", foram encontradas 12 diferentes aplicações, sendo que as principais formas de utilização das tecnologias de BI para apoio à gestão foram para a elaboração do orçamento das obras e para a gestão dos custos de obras em andamento. Além dessas, aplicações na área de gestão do conhecimento, gestão de riscos, gestão estratégica e em segurança do trabalho, gestão do cronograma, gestão da produtividade, gestão de estoques, gestão de fornecedores, gestão de resíduos e projeto estrutural foram evidenciadas. Com base nesses resultados, é possível aferir que as tecnologias de BI são utilizadas em distintos setores da Construção Civil, transcendendo a área de gestão financeira/executiva e apoiando até mesmo os processos de produção.

Em uma análise adicional que cruzou os dados de aplicação versus software utilizado, foi possível observar que 
para elaboração de orçamentos, gestão da produtividade, gestão de fornecedores, gestão de resíduos, gestão de riscos, gestão de custos, gestão do conhecimento, gestão estratégica e projeto estrutural, o uso de softwares desenvolvidos especificamente para cada empresa foi predominante. Já para as aplicações de gestão do cronograma e segurança do trabalho, o uso de softwares comerciais foi mais frequente.

Com isso, o presente trabalho apresenta diversas alternativas às empresas de construção civil para a adoção das tecnologias de BI. Entretanto, dificuldades técnicas e econômicas para o desenvolvimento de soluções personalizadas podem tornar a tarefa desafiadora. Segundo Lu et al. (2014), a falta de conhecimentos e habilidades sobre as ferramentas computacionais são obstáculos para a adoção das tecnologias no setor da construção civil, além disso, algumas empresas não utilizam essas ferramentas devido às incertezas sobre o retorno econômico gerado (Gajendran \& Brewer, 2012). Portanto, para superar esses obstáculos, poderão ser necessárias medidas de cooperação entre empresas, entidades de classe e universidades, para o desenvolvimento de pesquisas personalizadas para cada setor da indústria da construção.

Não foi possível observar nos estudos analisados a caracterização das empresas estudadas. Tal informação é importante, pois uma solução que pode ser útil para uma grande empresa pode não ser adequada para uma pequena construtora, e vice-versa. Além disso, em alguns estudos, foram propostos softwares que necessitam de testes em situações reais. Sugere-se que em trabalhos futuros sejam averiguados, por meio de estudos de caso, os desafios de implantação dessas ferramentas nas empresas, de acordo com as diferentes realidades organizacionais existentes, bem como as limitações na utilização das ferramentas.

Por fim, como os dados da revisão foram coletados antes da pandemia do novo Coronavírus, decretada pela Organização Mundial da Saúde em março de 2020, acredita-se que uma análise do uso dessas tecnologias no período pós-pandemia seja de grande interesse, pois as restrições à circulação de pessoas e às medidas de distanciamento social forçaram a adoção massiva de meios de comunicação digital, gerando assim um maior volume de dados produzidos e, consequentemente, maior necessidade de uso de ferramentas de análise de dados, como as de BI. 


\section{REFERÊNCIAS}

Ahmad, I., Azhar, S., \& Lukauskis, P. (2004). Development of a decision support system using data warehousing to assist builders/developers in site selection. Automation in Construction, 13(4), 525-542. doi: 10.1051/matecconf/201817001110.

Cao, Y., Chau, K. W., Anson, M., \& Zhang, J. (2002). An intelligent decision support system in construction management by data warehousing technique. In Engineering and deployment of cooperative information systems. edcis 2002. lecture notes in computer science (v. 2480, p. 19-42). Berlin: Springer. doi: doi.org/10.1007/3-540-45785-2_28.

Chau, K. W., Anson, M., \& Zhang, J. P. (2005). 4d dynamic construction management and visualization software: 1 . development. Automation in Construction, 14(4), 512-524. doi: 10.1016/j.autcon.20.11.002.

Chau, K. W., Cao, Y., Anson, M., \& Zhang, J. (2003). Application of data warehouse and decision support system in construction management. Automation in Construction, 12(2), 213-224. doi: 10.1016/S0926-5805(02)00087-0.

Chen, H., Chiang, R., \& Storey, V. (2002). Analyzing the past to prepare for the future: Writing a literature review $r$. MIS Quarterly, 26(2), 13-23. doi: 10.2307/4132319.

Chen, H., Chiang, R., \& Storey, V. (2012). Business intelligence and analytics: From big data to big impact. MIS Quarterly(36), 1165-1188. doi: 10.2307/41703503.

Cheng, C. W., Lin, C. C., \& Leu, S. S. (2010). Use of association rules to explore cause-effect relationships in occupational accidents in the taiwan construction industry. Safety Science, 48(4), 436-444. doi: 10.1016/j.ssci.2009.12.005.

Chong, H. Y., \& Phuah, T. H. (2013). Incorporation of database approach into contractual issues: Methodology and practical guide for organizations. Automation in Construction, 31(4), 512-524. doi: 10.1016/j.autcon.20.11.002.

Gajendran, T., \& Brewer, G. (2012). Cultural consciousness and the effective implementation of information and communication technology. Construction Innovation, 12(2), 179-197. doi: 10.1108/14714171211215930.

Girsang, A. S., Isa, S. M., Saputra, H., Nuriawan, M. A., Ghozali, R. P., \& Kaburuan, E. R. (2018). Business intelligence for construction company acknowledgement reporting system. In Proceedings of 1 st 2018 indonesian association for pattern recognition international conference, (p. 113-122). Indonésia: INAPR. doi: 10.1109/INAPR.2018.8627012.

Gowthami, S., \& Venkatakrishnakumar, S. (2016). Impact of smartphone: A pilot study on positive and negative effects. International Journal of Scientific Engineering and Applied Science, 3(2), 2395-3470. doi: 10.1016/j.autcon.20.11.002.

Hammad, A., AbouRizk, S., \& Mohamed, Y. (2014). Application of kdd techniques to extract useful knowledge from labor resources data in industrial construction projects. Journal of Management in Engineering, 30(6), 05014011. doi: 10.1061/(asce)me.1943-5479.0000280.

Instituto Brasilero de Geografia e Estatística. (2020). Contas nacionais trimestrais de 2019. Rio de Janeiro: IBGE.

Kitchenham, B. (2007). Guidelines for performing systematic literature reviews in software engineering. Biomedical and Environmental Sciences, 13(1), 37-43. doi: $10.1145 / 1134285.1134500$

Konikov, A. (2018). A selective study of information technologies to improve operations efficiency in construction.
MATEC Web of Conferences, 170(01110). doi: 10.1051/matecconf/201817001110.

Konikov, A., Kulikova, E., \& Stifeeva, O. (2018). Research of the possibilities of application of the data warehouse in the construction area. MATEC Web of Conferences, 251 (03062). doi: 10.1051/matecconf/201825103062.

Lu, Y., Li, Y., Skibniewski, M., Wu, Z., Wang, R., \& Le, Y. (2014). Information and communication technology applications in architecture, engineering, and construction organizations: A 15-year review. Journal of Management in Engineering, 31(1), 01-19. doi: 10.1061/(ASCE)ME.19435479.0000319 .

Ma, L., Luo, Bin, H., \& Chen, H. R. (2013). Safety risk analysis based on a geotechnical instrumentation data warehouse in metro tunnel project. Automation in Construction(34), 75-84. doi: 10.1016/j.autcon.2012.10.009.

Ma, Z., Lu, N., \& Wu, S. (2011). Identification and representation of information resources for construction firms. Advanced Engineering Informatics, 25(4), 612-624. doi: 10.1016/j.aei.2011.08.00.

Ma, Z., Lu, N., \& Wu, S. (2013). Web-based construction waste estimation system for building construction projects. Automation in Construction(35), 142-156. doi: 10.1016/j.autcon.2013.05.002.

Martínez-Rojas, M., Marin, N., \& Amparo Vila, M. (2012). The role of information technologies to address data handling in construction project management. Journal of Computing in Civil Engineering, 30(4), 01-11. doi: 10.1061/(ASCE)CP.1943-5487.

Martínez-Rojas, M., Marín, N., \& Miranda, M. A. V. (2016). An intelligent system for the acquisition and management of information from bill of quantities in building projects. Expert Systems with Applications (63), 284-294. doi: 10.1016/j.eswa.2016.07.011.

Montaser, A., \& Montaser, A. (2017). Web based project integrated controls system. In Proceedings of the $34 \mathrm{rd}$ isarc. Taipei, Taiwan: ISARC.

Moon, S. W., Kim, J. S., \& Kwon, K. N. (2007). Effectiveness of olap-based cost data management in construction cost estimate. Automation in Construction, 16(3), 336-344. doi: 10.1016/j.autcon.2006.07.008.

Muntean, M., \& Surcel, T. (2013). Agile bi: The future of bi. Informatica Economica, 17(3), 114-124. doi: 10.12948/issn14531305/17.3.2013.10.

Negash, S., \& Gray, P. (2003). Business intelligence. In Handbook on decision support systems 2. Berlin: Springer.

Rezaei, A. R., Çelik, T., \& Baalousha, Y. (2011). Performance measurement in a quality management system. Scientia Iranica, 18(3), 742-752. doi: 10.1016/j.scient.2011.05.021.

Rezgui, Y. (2001). Review of information and the state of the art of knowledge management practices in the construction industry. Knowledge Engineering Review, 16(3), 241-254. doi: $10.1017 /$ S026988890100008X.

Rujirayanyong, T., \& Shi, J. J. (2005). Companywide project data integration for a construction organization. Construction Research Congress 2005, 01-10. doi: 10.1061/40754(183)85.

Rujirayanyong, T., \& Shi, J. J. (2006). A project-oriented data warehouse for construction. Automation in Construction, 15(6), 800-807. doi: 10.1016/j.autcon.2005.11.001. 
Sapateiro, C., \& Rui, B. (2019). Business intelligence for construction company acknowledgement reporting system. In Proceedings of 11th ineka conference. Verona: INEKA. Recuperado de http://hdl.handle.net/10400.26/29147.

Szelka, J., \& Wrona, Z. (2010). Application of analytic databases to support decision making in structural engineering / zastosowanie analitycznych baz danych przy podejmowaniu decyzji w obszarze budownictwa ladowego. Archives of Civil Engineering, 56(2), 436-444. doi: 10.2478/v.10169010-0009-6.

Tranfield, D., Denyer, D., \& Smart, P. (2003). Towards a methodology for developing evidence-informed management knowledge by means of systematic review. British Journal of Management(14), 207-222.

Vuori, V. (2007). Business intelligence activities in construction companies in finland-a series of case studies. In Proceedings of the european conference on knowledge management (p. 1086-1092). Barcelona, Espanha: Curran Associates.

Wang, Q., Xi, L., \& Gao, K. (2009). Application of business intelligence in the information development of construction enterprise. In 5th international conference on natural computation, icnc 2009 (p. 212-215). Tianjin, China: ICNC. doi: 10.1109/ICNC.2009.674.

Como citar este artigo (APA):

Lopes, A. B., Boscarioli, C., Pereira, E. N. \& Bezerra, R. C. (2020). Business Intelligence para apoio à gestão na construção civil: uma revisão sistemática da literatura. AtoZ: novas práticas em informação e conhecimento, 9(1), 74 - 84. Recuperado de: http://dx.doi.org/10.5380/atoz.v9i1.72574 